\title{
LETTER
}

\section{Admission chest CT score predicts 5-day outcome in patients with COVID-19}

\author{
Elyas Mahdjoub $b^{1,2,3^{*}} \mathbb{D}$, Waqaas Mohammad ${ }^{1,3}$, Thomas Lefevre ${ }^{1,3}$, Marie-Pierre Debray ${ }^{1,3}$, Antoine Khalii ${ }^{1,2,3}$ \\ and Study Group $\$$
}

(c) 2020 Springer-Verlag GmbH Germany, part of Springer Nature

Dear Editor,

Since December 2019, COVID-19 has rapidly spread and 2,804,796 cases, including 193,710 deaths, have been reported worldwide as of April 26, 2020 [1]. While most patients have mild symptoms and recover without treatment, some patients may develop dyspnea, requiring oxygen therapy, or rapidly deteriorate into respiratory or multi-organ failure, requiring mechanical ventilation and intensive care unit monitoring [2]. Early identification of patients at risk of rapid worsening may help management of COVID-19 patients in emergency departments. CT is largely used for diagnosis of COVID-19, in addition to RT-PCR, and typically shows bilateral peripheral ground glass opacities with occasional consolidations [3]. The extent of lung lesions is correlated with disease severity [4]. However, data about the prognostic value of CT in COVID-19 are scarce [5]. We assessed an admission CT score for predicting the 5-day outcome in COVID-19 patients.

Patients with RT-PCR-confirmed COVID-19 who underwent CT from March 1 to March 20, 2020, at our institution were retrospectively enrolled. All CTs were

\footnotetext{
*Correspondence: elyas.mahdjoub@aphp.fr

${ }^{3}$ Hôpital Bichat-Claude-Bernard, 46 Rue Henri Huchard, 75018 Paris, France

Full author information is available at the end of the article
}

${ }^{\$}$ Study Group members: Alexandre Fitoussi: Radiology Department, Assistance Publique-Hôpitaux de Paris, Bichat-Claude Bernard University Hospital, Paris, France. Paul-Henri Wicky: Medical and Infectious Diseases Intensive Care Unit, Assistance Publique-Hôpitaux de Paris, Bichat-Claude Bernard University Hospital, Paris, France. Antoine Bachelard: Infectious and Tropical Diseases Department, Assistance Publique-Hôpitaux de Paris, Bichat-Claude Bernard University Hospital, Paris, France. Christophe Choquet:Emergency Department, Assistance Publique-Hôpitaux de Paris, Bichat-Claude Bernard University Hospital, Paris, France. independently scored by two radiologists blinded to clinical data. The CT score (range 0-25) was defined as the sum of lung involvement $(0: 0 \%, 1:<5 \%, 2: 5-25 \%$, 3: $25-50 \%, 4: 50-75 \%, 5:>75 \%)$ of each lobe, including ground glass opacity, crazy paving and consolidation (Fig. 1 and Supplementary Materials 1). Poor 5-day outcome was defined as need for mechanical ventilation or death within 5 days of the CT. Baseline characteristics were compared between patients with poor and good 5-day outcome (Supplementary Materials 2). The optimal cutoff CT score for discriminating patients with poor and good outcome was determined using ROC curve (Supplementary Materials 3) and maximizing the Youden index. To identify predictors for poor 5-day outcome, baseline characteristics with $p$ value $<0.10$ were entered into multivariate logistic regression (Supplementary Materials 4). Inter-observer reliability for CT score was assessed. Institutional review board approval was obtained and patient consent was waived.

142 COVID-19 patients were enrolled, of whom 12 (8.5\%) required mechanical ventilation and $8(5.6 \%)$ died within 5 days of the CT (Supplementary Materials 5). Patients with poor 5 -day outcome had higher median CT scores (15; interquartile range 13-20) than other patients (8; 5-11, $p<0.001)$. The optimal CT score to predict poor 5 -day outcome was $\geq 13$ (sensitivity $80 \%$, specificity $85.2 \%$, AUC 0.853 ). On multivariate analysis, CT score $\geq 13$ was related to poor 5 -day outcome (odds ratio 44.243, 95\% CI 8.609-227.365, $p<0.001)$. Inter-observer reliability was excellent (intraclass correlation coefficient $0.976,95 \%$ CI $0.966-0.983)$.

We found that the admission CT score was an independent predictor for 5-day outcome of COVID19 patients. Our results are consistent with those of Colombi et al. [5] who showed that well-aerated lung

\section{望



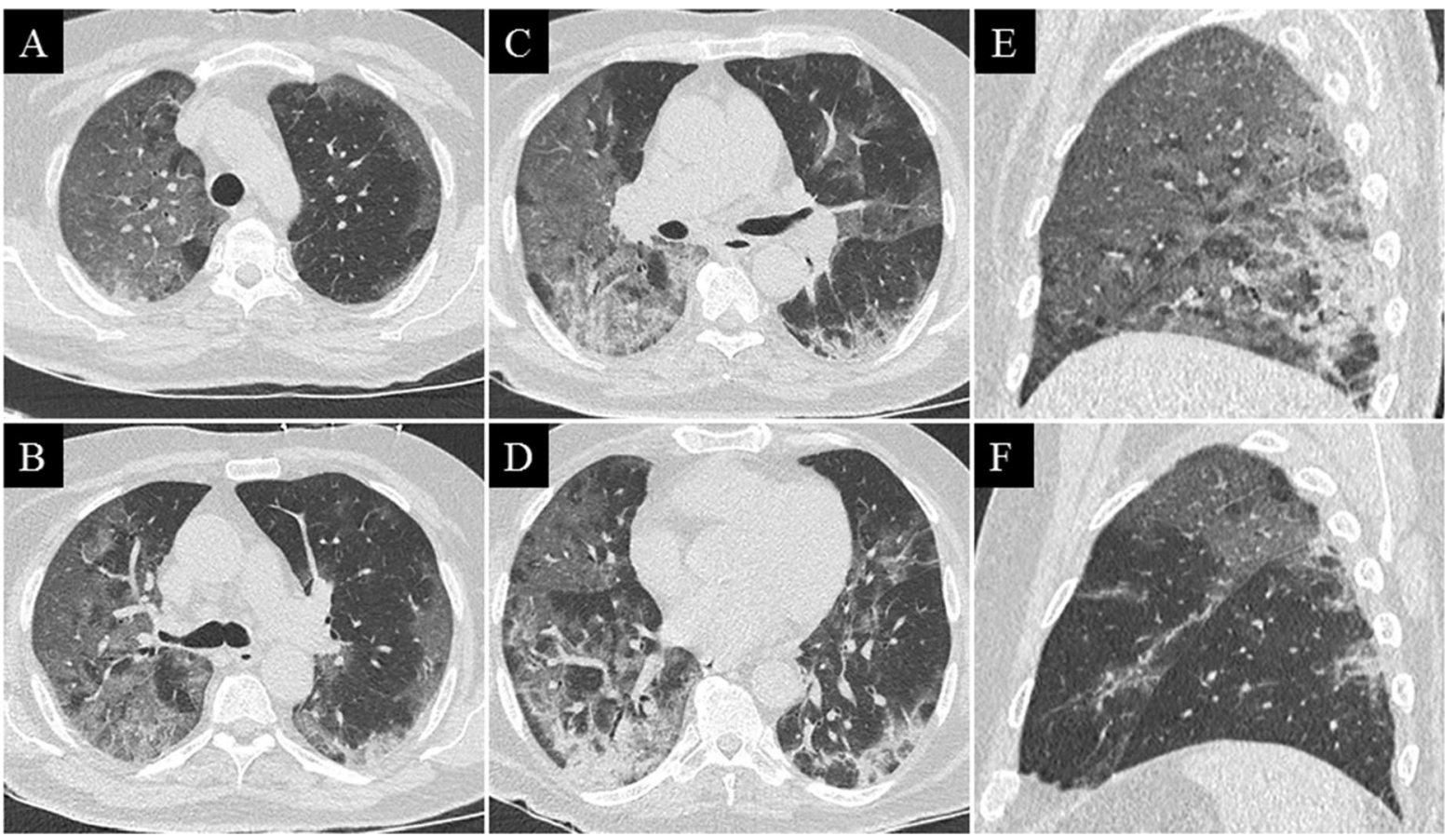

Fig. 1 Axial and sagittal CT images from a 63-year-old man presenting at emergency room for severe COVID-19 at day 12 after symptom onset. Axial (a-d), right (e) and left (f) sagittal selected CT images showed extensive, bilateral and asymmetric ground glass opacities involving almost the entire right lung, with small consolidation in the right lower lobe. Left lung involvement was less severe. The lobe score was 5 (>75\%) for right upper lobe, 5 (> 75\%) for right middle lobe, 5 (> 75\%) for right lower lobe, 3 (25-50\%) for left upper lobe and 2 (5-25\%) for left lower lobe. The tota CT score for this patient was 20. The patient presented respiratory failure requiring mechanical ventilation 1 day after this scan

quantification predicted intensive care unit admission or death. Interestingly, the CT score is simple, reproducible and readily available in daily practice with no need of post-processing software. It may help select patients at risk of rapid deterioration who should require high-level monitoring. External validation of our non-replicated single-center results and comparison of CT score with $\mathrm{X}$-ray or ultrasound-based scores are needed.

\section{Electronic supplementary material}

The online version of this article (https://doi.org/10.1007/s00134-020-06118-y) contains supplementary material, which is available to authorized users.

\section{Author details}

${ }^{1}$ Radiology Department, Assistance Publique-Hôpitaux de Paris, Bichat-Claude Bernard University Hospital, Paris, France. ${ }^{2}$ Paris University, Paris, France.

${ }^{3}$ Hôpital Bichat-Claude-Bernard, 46 Rue Henri Huchard, 75018 Paris, France.

\section{Author's contributions}

Each author has participated sufficiently in the work to take public responsibility for the content, including a substantial contribution to conception and design, execution, or analysis and interpretation of data. Elyas Mahdjoub was involved in literature search, figures, study design, data collection, data analysis, data interpretation, and writing. Waqaas Mohammad was involved in literature search, figures, data analysis, and data interpretation. Thomas Lefevre was involved in literature search, data collection, data analysis, and data interpretation. Alexandre Fitoussi was involved in literature search, study design, data collection, and data analysis. Paul-Henri Wicky was involved in study design, and data collection. Antoine Bachelard was involved in study design and data collection. Christophe Choquet was involved in study design and data collection. Marie-Pierre Debray was involved in study design, data collection, data analysis, and data interpretation. Antoine Khalil was involved in literature search, figures, study design, data collection, data analysis, data interpretation, and writing. All authors were involved in drafting the article or revising it critically for important intellectual content, and have read and approved the final version of the manuscript.

\section{Compliance with ethical standards}

\section{Conflicts of interest}

None.

\section{Publisher's Note}

Springer Nature remains neutral with regard to jurisdictional claims in published maps and institutional affiliations.

Accepted: 13 May 2020

Published online: 28 May 2020

\section{References}

1. WHO. Coronavirus disease (COVID-2019) situation reports. https://www. who.int/emergencies/diseases/novel-coronavirus-2019/situation-reports. Accessed on 27 April 2020

2. Wang D, Hu B, Hu C et al (2020) Clinical characteristics of 138 hospitalized patients with 2019 novel coronavirus-infected pneumonia in Wuhan, China. JAMA 323:1061. https://doi.org/10.1001/jama.2020.1585 
3. AiT, Yang Z, Hou H et al (2020) Correlation of chest CT and RT-PCR testing in coronavirus disease 2019 (COVID-19) in China: a report of 1014 cases. Radiology. https://doi.org/10.1148/radiol.2020200642

4. Yang R, Li X, Liu H et al (2020) Chest CT severity score: an imaging tool for assessing severe COVID-19. Radiol Cardiothorac Imaging 2:e200047. https ://doi.org/10.1148/ryct.2020200047
5. Colombi D, Bodini FC, Petrini M et al (2020) Well-aerated lung on admitting chest $\mathrm{CT}$ to predict adverse outcome in COVID-19 pneumonia. Radiology. https://doi.org/10.1148/radiol.2020201433 